\title{
Avaliação das metodologias brasileiras de vulnerabilidade socioambiental como decorrência da problemática urbana no Brasil
}

\author{
Analysis of the Brazilian assessment methodologies of socio- \\ -environmental vulnerability as a result of urban problems in Brazil
}

Mônica Maria Souto Maior Gesinaldo Ataíde Cândido

\begin{abstract}
Resumo
0 artigo intenta refletir e analisar as principais metodologias de avaliação da vulnerabilidade socioambiental propostas e aplicadas em contextos específicos no Brasil, através de um ensaio teórico-comparativo utilizando um conjunto de critérios de avaliação retiradas das variáveis existentes nos diversos modelos pesquisados. Os resultados apontam que os modelos foram contributivos para o avanço dos estudos da vulnerabilidade socioambiental no Brasil, possibilitando um diagnóstico preciso dos fatores que contribuem para acentuar e mitigar o fenômeno. No entanto, dadas a dinâmica e complexidade do processo de urbanização e suas implicações, se fazem necessárias constantes adaptações das metodologias criadas, assim como a criação de novas metodologias que consigam traduzir fidedignamente a dinâmica e complexidade da urbanização, sobretudo para as comunidades mais carentes.
\end{abstract}

Palavras-chave: processo de urbanização; vulnerabilidade socioambiental; metodologias; avaliação e indicadores.

\begin{abstract}
The article intends to reflect on and analyze the main methodologies to assess socio-environmental vulnerability that have been proposed and applied to specific contexts in Brazil, through a comparative theoretical essay using a set of assessment criteria extracted from the variables existing in the diverse surveyed models. The results show that the models have contributed to the advancement of socio-environmental vulnerability studies in Brazil; in addition, they have enabled an accurate diagnosis of the factors that contribute to intensify and mitigate the phenomenon. However, given the dynamics and complex nature of the urbanization process and its implications, it is necessary to constantly adapt the established methodologies, as well as to create new methodologies that are able to translate faithfully the dynamics and complexity of urbanization, particularly for poor communities.
\end{abstract}

Keywords: urbanization process; socioenvironmental vulnerability; methodologies; assessment and indicators. 


\section{Introdução}

No último século, houve uma diminuição dos índices de pobreza na América Latina, mesmo assim os modelos que estudam a população em vulnerabilidade continuam sendo instrumentos eficazes de análise da situação dos excluídos latino-americanos, porque são capazes de identificar as características de comportamento individuais e as mudanças repentinas de ascensão e declínio social, as quais vão além das questões ligadas à renda.

A preocupação dos estudos que envolvem a vulnerabilidade socioambiental, em contexto urbano latino-americano, é oferecer um painel sobre os fatores socioambientais que influenciam e são influenciados pela fixação da população pobre em áreas de risco, as quais podem gerar danos. Dessa forma, a vulnerabilidade socioambiental urbana está vinculada, também, aos fenômenos de adensamento populacional, à segregação espacial urbana, aos processos de exclusão social e às injustiças ambientais, processos ligados diretamente ao aumento demográfico e à falta de políticas públicas eficazes.

Especificamente no contexto geográfico brasileiro, desde o século passado, se tem presenciado um aumento demográfico urbano substantivo, o que traz consequências diretas na estruturação e ordem das principais cidades brasileiras, desregulando o sistema socioambiental, expondo a população citadina a uma situação crescente de vulnerabilidade, principalmente nas áreas centrais de preservação ou nas periféricas, onde os fatores de riscos ambientais se tornam desastrosos diante da instabilidade socioeconômica da população pobre.
Assim, a vulnerabilidade pode ser entendida como um processo gerado por diversos fatores socioambientais, os quais, em conjunto, fragilizam pessoas, gerando consequências desastrosas como perdas materiais e/ou de vida.

Fundamentada em tais teorias, o termo "vulnerabilidade socioambiental" começou a ser construído entre as décadas de oitenta e noventa, quando pesquisadores a exemplo de D’Ercole (1994), Blaikie et al. (1994), Fournier (1995), Cardona (1996), Hewitt (1997), Moser (1998), Kaztman et al. (1999), Gonzáles et al. (1999), dentre outros, avaliaram a importância do significado das condições sociais na incidência, extensão e distribuição das ameaças naturais. Eles mostraram que a perda e a sobrevivência estão relacionadas muito de perto com os padrões e as variações da qualidade de vida material da sociedade, tanto no que se refere à ocorrência de danos e tipos estabelecidos, e onde, como e especialmente a quem afetam.

Segundo Cardona (1996), os danos materiais dependem especialmente do uso da terra, padrões de assentamento e da concepção e localização de estruturas construídas, e esses danos são desproporcionalmente concentrados em determinados grupos etários, de acordo com sexo ou ocupação, níveis de renda e da falta de voz política do povo.

Na América Latina e nos países em desenvolvimento, devido a uma estrutura política e econômica em constante crise, a ideia de estabilidade não é observada. Essa conjuntura dificulta a geração de novas frentes de trabaIho, aumentando a instabilidade socioeconômica das famílias, associando a vulnerabilidade à ideia da falta de oportunidades existente diante do desemprego, da precariedade do trabaIho, da pobreza, da falta de proteção social e 
da fragilidade das relações sociais, problemas que afetam a todos de um modo geral.

Adicionados aos problemas citados anteriormente, as cidades brasileiras apresentam uma concentração da população de baixa renda de forma desigual, numa mesma extensão geográfica, onde se observa a ocorrência de eventos naturais como enchentes, deslizamentos, desmoronamentos e/ou vendavais, que causam perdas e danos de toda ordem (Deschamps, 2004).

Diante deste quadro, diversos pesquisadores brasileiros vêm desenvolvendo modelos para estudar a vulnerabilidade socioambiental: Deschamps (2004; 2006), Hogan (2007), Alves (2010a), Almeida (2010) e Alves et al. (2010b), os quais trabalham as famílias expostas aos riscos socioeconômicos e ambientais. Esses cinco modelos brasileiros trazem diferentes ferramentas de abordagem, e cada uma delas foi aplicada considerando fatores ambientais específicos para cada espaço geográfico estudado.

Nessa perspectiva, busca-se, por meio deste ensaio teórico, comparar essas cinco metodologias, utilizando-se os seguintes critérios de análise: características do método, campo geográfico de atuação, dimensões mensuradas, variáveis trabalhadas, tratamento dos dados e vínculos com grupos de pesquisa, os quais foram escolhidos para introduzir uma análise mais profícua das aplicações e abrangência dos indicadores utilizados para a realidade socioambiental brasileira.

Em termos metodológicos, o artigo pode ser classificado como um ensaio teórico formal, para cujo estudo, depois da identificação dos principais estudos e pesquisas realizados no Brasil, foi estabelecida a seguinte sequência metodológica: 1) levantamento de todas as metodologias brasileiras que estudaram o fenômeno da vulnerabilidade socioambiental até 0 ano de $2010 ; 2$ ) identificação dos fatores estudados em cada metodologia, considerando as dimensões e indicadores; 3 ) compilação dos indicadores, buscando identificar sua reincidência nos modelos; 4) comparação das principais características; e 5) reflexões sobre a profundidade de abrangência dos modelos para o contexto brasileiro. Com isso, foi possível identificar uma série de variáveis similares e diferentes utilizadas para situações e contextos diferenciados e, a partir disso, constatando-se também que tais variáveis poderiam ser utilizadas como marco ordenador para novos estudos e pesquisas relacionados ao tema vulnerabilidade socioambientais de comunidades em espaços urbanos.

Além deste conteúdo introdutório, o artigo apresenta, nos seus demais itens, uma fundamentação teórica sobre vulnerabilidade; os resultados, considerando os aspectos relacionados aos conceitos e teorias sobre as metodologias da vulnerabilidade socioambiental estudadas; comparação das metodologias; e as conclusões geradas por este estudo.

\section{Fundamentação teórica}

\section{Vulnerabilidade}

0 conceito de vulnerabilidade está correlacionado a uma construção teórica, anterior a ela, definida como exclusão social, que serviu de referência para a caracterização de situações sociais-limite, de pobreza ou marginalidade, e para a consequente formulação de políticas 
públicas voltadas para o enfrentamento destas questões (Dieese, 2007; Busso, 2005; Lavinas, 2002; Castel, 1997).

Dessa forma, é importante ressaltar a semelhança espacial, histórica e conceitual que envolve a interligação entre esses dois termos. A exclusão social teve sua origem na França, no século $X X$, e se estendeu a outros países europeus para ressaltar situações que iam além do mercado de trabalho e que representavam rupturas de vínculos sociais e perdas da base de sustentação da reprodução da vida: a casa, a vizinhança e a família (Castel, 1997).

Diante dessa conjuntura e como parte de um mesmo campo conceitual, há os que relacionam a perda do vínculo social como resultante da perda de solidariedade e aqueles que a vinculam à negação dos direitos sociais estabelecidos. Segundo Kowarick (2003), o interesse pelo campo da exclusão na Europa surge em virtude de uma situação de mudanças tecnológicas, reestruturação econômica e desmantelamento do estado de bem-estar social, em que o estado de exclusão caracterizaria um conjunto de situações marcadas pela falta de acesso aos meios de vida e que afetaria a plena integração social até então existente.

Segundo Castel (1997), para se chegar numa situação de exclusão social, é necessário passar por três estágios distintos: uma etapa inicial de integração social, em que se teria uma situação de estabilidade econômica e social; um momento crítico de vulnerabilidade caracterizada pela precariedade do trabalho e a fragilidade dos apoios proporcionados pelas relações familiares e sociais; e, finalmente, a chegada ao estágio final - o de exclusão social. Nessa visão, a vulnerabilidade identificaria a fragilidade do vínculo social antes de sua ruptura. Afirma ainda que não somente a falta de recursos materiais define os grupos como vulneráveis, mas também a instabilidade de suas relações sociais, que os fragiliza.

Kaztman (1999; 2000) analisa a vulnerabilidade a partir da existência, ou não, por parte dos indivíduos ou das famílias, de ativos disponíveis e capazes de enfrentar determinadas situações de risco. Ele trabalha o conceito de capital para os grupos vulneráveis, que pode capacitá-los a aproveitar as oportunidades disponíveis em distintos âmbitos socioeconômicos, e que influencia o estado de respostas diante das situações de risco.

Observa-se que os estudos de Kaztman não consideram as estruturas de oportunidades como um fator constante, ao contrário, essas estruturas variam de acordo com a área geográfica e com os fatores temporais históricos. Ele incorpora na sua ideia de ativos/vulnerabilidade/estrutura de oportunidades o conceito de mobilidade social, como fator determinante das situações de ascensão e declínio da condição de vulnerabilidade. Segundo o Dieese (2007), essa noção de vulnerabilidade social, que considera a relação ativos/vulnerabilidade/estrutura de oportunidades, tem sido adotada para a construção de indicadores sociais mais amplos, não se restringindo à delimitação de uma determinada linha de pobreza.

Blaikie et al. (1994) afirma que a vulnerabilidade está diretamente associada à capacidade de um grupo ou família para resistir a efeitos nocivos e perigo e de se recuperar facilmente. Assim, a vulnerabilidade envolve uma combinação de fatores que determina o grau em que a vida de alguém ou de um grupo é colocada em risco por um evento discreto e 
identificável (ou uma série de tais eventos), na natureza e na sociedade.

Segundo Hewitt (1997) e Lavell (2000), as ameaças naturais, a destruição e sua distribuição social e territorial podem tornar o evento físico como um ponto de referência, mas, no final, a perda é determinada pelas diferenças de níveis de exposição e vulnerabilidade da população, infraestrutura e produção. Devido a essa enorme variedade entre diferentes espaços e unidades sociais, será consequentemente diferenciada a capacidade dos indivíduos em se recuperar, porque, mesmo dentro de um único nível de unidade espacial ou social, serão encontrados diferentes níveis de danos que refletem essa estruturação heterogênea da vulnerabilidade social.

D’Ercole (1994) e Blaikie et al. (1994) estabelecem uma relação de causa e efeito gerada entre a natureza e a sociedade, reconhecendo que os fatores de risco estão associados a um certo grau de exposição a uma situação crítica, natural ou social, que gera vulnerabilidade em determinados grupos; essas contextualizações incorporam ao fenômeno da vulnerabilidade uma perspectiva temporal de futuro, quando estabelecem que os grupos mais vulneráveis são também aqueles que possuem mais dificuldades para reconstruir suas vidas após algum desastre; consequentemente, esses mesmos grupos se tornarão mais vulneráveis aos efeitos de desastres futuros.

Kaztman (1999) concorda com esses dois autores quando considera que uma má resposta a um evento potencialmente danoso está relacionada ao gradiente de vulnerabilidades sociais e econômicas dos indivíduos ou grupos diante do evento, e que suas condições precárias de habitação, inadequados ativos de recursos humanos no seio das famílias, alimentação insuficiente e de má qualidade, alta permeabilidade aos serviços sociais, controle deficiente aos cuidados de saúde, falta de redes de reciprocidades e contatos, são alguns dos fatores que determinam o grau dessa vulnerabilidade. Segundo Cardona (1996), no contexto urbano, as zonas de riscos coincidem com áreas que apresentam condições de marginalidade ou subnormalidade, e seus habitantes têm níveis de renda que impossibilitam seu acesso a instituições de crédito para habitação, quando esse benefício de crédito existe.

Essas teorias abordadas mantêm uma conexão entre o ambiental e o social, as quais exercem intrínseca influência no meio urbano sobre uma comunidade, grupo social ou famílias e, assim, essa conexão socioambiental influencia o modo de resposta diante de situações que geram vulnerabilidade.

D'Ercole (1994) afirma que a análise da vulnerabilidade na cidade não pode deixar de contar com uma abordagem sistêmica que inclua: fatores socioeconômicos (êxodo rural e especulação imobiliária), fatores psicossociológicos (memória de risco, percepção e cultura de risco), fatores ligados à cultura e à história das sociedades expostas (autoconstrução, lançamento de dejetos), fatores técnicos (prevenção), fatores funcionais (gestão de crise) e fatores institucionais (gestão de risco).

$\mathrm{Na}$ cidade, alguns desses fatores são elementos inerentes ao crescimento urbano, e fortemente integrados à dinâmica urbana, principalmente em países em desenvolvimento, onde há ausência de controle, má qualidade da infraestrutura, falta de planejamento e legislação urbana ineficiente, permitindo a expansão urbana para áreas de preservação e/ou de risco. 


\section{Vulnerabilidade socioambiental}

Segundo Deschamps (2004), Alves et al. (2008) e Almeida (2010), o quadro teórico, no qual se insere a vulnerabilidade socioambiental urbana, contempla a sobreposição (coexistência espacial) dos processos de expansão urbana envolvendo tanto a dispersão espacial de grupos de risco social, degradação ambiental e falta de serviços de infraestrutura urbana. Dessa forma, não se pode tratar da vulnerabilidade socioambiental sem considerar a expansão urbana para áreas periféricas, relacionada à procura por habitação em áreas com baixo valor da terra e sem infraestrutura. Essa dinâmica da expansão urbana, para regiões periféricas e periurbanas, estabelece uma condição de ocupação dos pobres e miseráveis de residir em áreas com más condições urbanísticas e de infraestrutura - sem abastecimento de água tratada, sem saneamento, sem coleta de lixo, etc. -, tais como: terrenos com alta declividade ou próximos a cursos d'água e de lixões, geralmente áreas públicas e/ou de preservação. Os índices de pobreza quantificam o grau da exclusão que fatores socioeconômicos impõem em um determinado lugar a alguns grupos.

0 nível de vulnerabilidade em que as famílias estão expostas aos riscos está vinculado à capacidade de respostas e ajustes diante das condições adversas ao meio, seja pela capacidade de mobilizar ativos para enfrentar as adversidades, por pouco capital humano ou pouco acesso à informação, ou seja, pelas poucas habilidades sociais básicas, com falta de relações pessoais e com pouca capacidade para manejar recursos (Deschamps, 2006).

A vulnerabilidade deve considerar algumas dimensões que subsidie as análises dos riscos e ameaças dentro do seu sistema. Segundo Fournier (1985), a diferença fundamental entre o risco e a ameaça é que a ameaça está relacionada com a probabilidade de que se manifeste um evento natural ou um evento provocado, enquanto o risco está relacionado com a probabilidade de que se manifestem certas consequências, que estão estreitamente relacionadas, não só com a extensão da vulnerabilidade da exposição dos elementos sujeitos, mas ainda com a certeza que esses sujeitos têm de ser afetados pelo acontecimento. Nesse contexto, a vulnerabilidade pode ser entendida como a predisposição intrínseca de um sujeito ou elemento a sofrer danos, devido à possibilidade de ações externas e, portanto, sua avaliação contribui fundamentalmente para o conhecimento do risco por meio de interações do elemento suscetível com o ambiente perigoso (Cardona, 1996).

Cutter (2003) afirma que está embutido em toda a discussão sobre a ciência da vulnerabilidade socioambiental o requisito de antecipar surpresa, capturar a incerteza e adaptar-se às mudanças, salientando que se precisa investir ainda muito no conhecimento sobre essa ciência, havendo a necessidade de conectá-la a um campo teórico mais amplo e a uma arena de ação política comprometida com a justiça social e ambiental. Ela ainda promove a necessidade de uma confluência dos conhecimentos sobre as dinâmicas sociais e naturais, condição imprescindível para um diagnóstico e um prognóstico. Assim, a ciência da vulnerabilidade evoca uma visão multidimensional associada a seus fenômenos geradores nos processos de distribuição, gestão e experiências dos riscos, ameaças e vulnerabilidades. 
Diante do que se expôs sobre as peculiaridades geográficas, temporais, socioeconômicas e dos fenômenos a serem estudados, pesquisadores brasileiros como Alves (2008), Deschamps (2004; 2006), Almeida (2010) e Silveira (2010) trabalham com as seguintes dimensões em relação à vulnerabilidade das famílias expostas aos riscos num contexto de expansão urbana: econômicos, sociais e ambientais. Os autores ainda descrevem características demográficas, que devem ser consideradas na unidade doméstica e que tendem a acentuar a vulnerabilidade: estrutura familiar, ciclo de vida e aspectos demográficos. Nesse contexto social, as indagações partem da necessidade de resposta sobre quais os elementos que mais contribuem para a vulnerabilidade social e se esses elementos afetam de forma homogênea os diferentes grupos sociais. No contexto ambiental, os principais aspectos considerados por esses autores são os relacionados à infraestrutura urbana, considerando os danos que sua falta pode trazer em termos de saúde e de qualidade de vida.

Os estudos que abarcam a vulnerabilidade buscam contribuir para avaliação das diferenças socioambientais urbanas, porque abrangem todo o sistema atingido pelo adensamento populacional. Dessa forma, conhecer os modelos utilizados, suas ferramentas e seu conjunto de indicadores, pode apontar a estreita relação entre a segregação social urbana, o sistema de infraestrutura urbana e o processo de adensamento. Esse conhecimento servirá para vislumbrar soluções específicas em cada localidade, porque as cidades apresentam problemas específicos diante do fenômeno de transbordamento urbano. Sendo assim, se faz necessário conhecer os procedimentos adotados em cada um dos modelos brasileiros, para conhecer o quadro da sistemática de avaliação da vulnerabilidade socioambiental para a realidade brasileira.

\section{Apresentação e análise dos resultados}

\section{Apresentação das metodologias estudadas}

Nos meios científicos brasileiros, o estudo da vulnerabilidade socioambiental é tratado em âmbito local, identificando grupos populacionais submetidos a um alto grau de risco em relação a desastres específicos de países em desenvolvimento. Nos últimos dez anos, as pesquisas avançaram, e foram criadas e aperfeiçoadas metodologias com o duplo objetivo de entender como o processo de adensamento populacional e expansão urbana influenciava e influencia a situação de risco de forma desigual a grupos populacionais específicos. Dentre elas, cinco se destacam por sua qualidade metodológica, pelo impacto gerado e pelo campo de pesquisa aberto nos meios científicos.

Dentre os modelos analisados todos partem do método dedutivo, que faz uso do raciocínio, a partir de fatos e indícios, para obter uma conclusão a respeito de determinadas premissas. No caso da vulnerabilidade socioambiental, os níveis de risco e vulnerabilidade são estudados tomando por base a identificação de relações estatísticas significativas dentre um conjunto de potenciais indicadores, estabelecendo relações com uma proposição geral para, a partir de raciocínio lógico, chegar à verdade daquilo que se propõe. 
Todos os modelos usam um mesmo procedimento metodológico, baseado em dados do IBGE e da sobreposição cartográfica dos riscos ambientais com os riscos sociais distribuídos no espaço urbano estudado, utilizando a análise multivariada, correlação de indicadores (matriz de correlação de Pearson) - Método de agrupamento não hierárquico das k-médias ou método do núcleo de Kernel.

\section{Modelo de Alves}

A pesquisa de Alves et al. (2010a) foi resultado de um projeto desenvolvido em parceria com o Centro de Estudos da Metrópole (CEM Cebrap) e a Coordenadoria de Observação da Terra do Instituto Nacional de Pesquisa Espaciais (OBT-Inpe). 0 objetivo do trabalho é fazer uma análise, em escala intraurbana, das interrelações entre processos de expansão urbana e situações de vulnerabilidade socioambiental do distrito de Cidade Tiradentes e seu entorno, no extremo leste do município de São Paulo.

Desenvolvida no período de 2000 a 2006, considerando as dimensões sociais e ambientais das dinâmicas de urbanização que estão ocorrendo na região hiperperiférica metropolitana de São Paulo, o foco da pesquisa recai nos processos de expansão urbana e nas situações de vulnerabilidade socioambiental, tendo como pressuposto que a presença de populações de baixa renda em áreas sem infraestrutura, serviços urbanos e com risco de degradação ambiental podem gerar novas situações de vulnerabilidade socioambiental.

A metodologia utilizada para o desenvolvimento da pesquisa partiu do levantamento cartográfico das áreas de risco socioeconômico da população, considerando estudos anteriores baseados no Censo Demográfico do IBGE 2000, que já haviam determinado as áreas de alta vulnerabilidade social (para fins deste estudo as áreas de baixa vulnerabilidade social foram descartadas). Em seguida, foi feito um cruzamento dessa população com as áreas de alta e baixa vulnerabilidade ambiental, considerando nesta dimensão o tipo de uso do solo urbano como: assentamentos precários ou não, favelas, conjuntos habitacionais, residencial consolidado, áreas urbanizadas e loteamentos irregulares. Em seguida, fez-se o cruzamento dos dados através de análise quantitativa - em termos de área e percentagem de área - da inserção da população de alta vulnerabilidade social nas áreas de baixa e alta vulnerabilidade ambiental.

Os resultados são apresentados num quadro que mostra uma classificação tipológica de uso do solo urbano em três tipos: assentamentos não precários - constituído de conjunto habitacional e residencial consolidado; assentamentos precários - constituído de favelas e loteamentos irregulares; e áreas urbanizadas - constituídas de indústrias, comércios e instituições. Essas áreas são classificadas percentualmente e em número de quilômetros quadrados, em dois tipos de vulnerabilidade socioambiental: baixa e alta.

0 diagnóstico é feito pela comparação evolutiva da mesma área em períodos diferentes, baseado nas percentagens do crescimento de assentamentos não precários, precários e da área urbanizada, confrontados com as áreas de baixa e alta vulnerabilidade ambiental, sendo considerada a mais crítica aquela que apresenta um crescimento de assentamentos precários em áreas de alta vulnerabilidade 
ambiental. Os resultados são visualizados em mapas cartográficos.

\section{Metodologia de Alves}

A pesquisa de Alves et al. (2010b) foi desenvolvida, no contexto urbano do Litoral Paulista, formada de 16 municípios, para identificar áreas com alta vulnerabilidade às mudanças climáticas, permitindo assim a construção de indicadores em escala desagregada, que representem as dimensões da vulnerabilidade - susceptibilidade e exposição ao risco ambiental com a integração de dados socioeconômicos, demográficos e ambientais. A pesquisa parte da hipótese de que os problemas recorrentes, associados a eventos extremos causados pela mudança climática, estão relacionados a ocupações irregulares em encostas ou nas margens dos corpos de água, falta de abastecimento de água potável para toda a população e falta de saneamento básico.

A pesquisa explora a relação entre população e meio ambiente, buscando a identificação e caracterização das áreas de maior risco e dos grupos populacionais mais vulneráveis às mudanças climáticas nas áreas urbanas, utilizando conjuntos específicos de variáveis socioeconômicas e ambientais.

0 procedimento estatístico adotado como técnica de análise foi baseado na estimativa da densidade de Kernel, que é um método não paramétrico para estimação de curvas de densidades, em que cada observação é ponderada pela distância em relação a um valor central, o núcleo. A ideia é centrar cada observação " $x$ " onde se queira estimar a densidade, uma janela " $b$ " que define a vizinhança de " $x$ " e os pontos que pertencem à estimação, ou seja, é uma técnica de análise espacial que se baseia na criação de superfícies de densidade.

Essa estimativa é apropriada para posições de dados individuais; entretanto, pode-se adotar esta técnica se o interesse é mostrar regiões menos fragmentadas de um determinado evento ou conjunto de eventos (Alves, 2010b).

0 método pode ser descrito da seguinte forma: se "s" representa uma localização qualquer numa região "R" e "s1", ..., "sn" são as localizações dos " $n$ " eventos observados, então a intensidade $\lambda($ s), é estimada por:

$$
\lambda_{\tau}(s)=\frac{1}{\delta(s)} \sum_{i=1}^{n} \frac{1}{\tau^{2}} \mathrm{k}\left(\frac{s-s i}{\tau}\right)
$$

em que "k" é uma função de densidade bivariada escolhida, conhecida como Kernel, e T o raio de influência. Para isso, foi considerada cada unidade de setor censitário como unidade de análise, na qual foi estimada a densidade de eventos segundo o centróide de cada setor censitário. Assim, a distribuição de eventos foi transformada em uma superfície contínua de vulnerabilidade socioambiental no litoral paulista, onde as áreas mais vulneráveis são indicadas pelas zonas de cores mais escuras nos mapas cartográficos.

\section{Metodologia de Almeida}

0 estudo desenvolvido por Almeida (2010) trata de pesquisa que explora as vulnerabilidades socioambientais de rios urbanos na Região Metropolitana de Fortaleza/CE. Esse estudo parte da hipótese de que há uma coincidência entre 
espaços susceptíveis a processos naturais perigosos, neste caso inundações, com espaços da cidade que apresentam os piores indicadores sociais, econômicos e de acesso a serviços e infraestrutura urbana. Teve como objetivo analisar os riscos e as vulnerabilidades socioambientais de rios urbanos no Brasil, tendo a bacia hidrográfica do Rio Maranguapinho, localizada na Região Metropolitana de Fortaleza - RMF, Ceará, como área de estudo de caso para compreensão das interrelações das vulnerabilidades sociais e exposição aos riscos naturais, principalmente as inundações.

Essa pesquisa foi desenvolvida em 2010 e chegou-se um índice de vulnerabilidade socioambiental através da sobreposição de dois índices: de vulnerabilidade social e de vulnerabilidade físico-espacial às inundações. Dessa forma, foram utilizados dados secundários do IBGE do Censo Demográfico de 2000, de acordo com variáveis que caracterizam amplas dimensões e desvantagens sociais e que correspondem a fatores recorrentes utilizados pelas ciências sociais.

A pesquisa trabalha com dois tratamentos diferenciados de acordo com a dimensão pesquisada: social e ambiental. No tratamento fornecido à dimensão social, foi realizada uma compilação através da junção de duas ou mais variáveis do Censo 2000, resultando, de 59 indicadores, apenas 21.

Para análise estatística dos dados, inicialmente realizou-se análise fatorial das variáveis. 0 procedimento é uma técnica estatística multivariada que, de acordo com a estrutura de dependência existente entre as variáveis de interesse (matriz de correlações ou covariâncias entre as variáveis), permite a redução da quantidade de variáveis para fatores que explicam um percentual representativo da variabilidade total das variáveis em estudo. Nesta pesquisa, os resultados da análise fatorial basearam-se na matriz de correlação entre as respostas dos itens.

Após a determinação das cargas fatoriais de cada indicador, foi estimado para cada setor censitário o valor correspondente de cada fator, sendo possível verificar a situação desses setores em relação à vulnerabilidade associada aos quatro fatores descritos a seguir: a) fator 1 - está relacionado à vulnerabilidade decorrente da educação; b) fator 2 está relacionado à vulnerabilidade decorrente das condições de infraestrutura e habitação; c) fator 3 - relacionado à vulnerabilidade em virtude do contingente populacionalde idosos (maiores de 64 anos); e d) fator 4 - relacionado à vulnerabilidade decorrente do contingente populacional de jovens (faixa etária de 10 a 19 anos).

Foi possível, ao final, dividir esses setores censitários de acordo com a média dos fatores sendo esses assim classificados: 1) vulnerabilidade social muito alta; 2) vulnerabilidade social alta; 3) vulnerabilidade social média a alta; 4 ) vulnerabilidade social média a baixa; 5) vulnerabilidade social baixa; e 6) vulnerabilidade social muito baixa. 0 intervalo para esse estudo, das médias dos fatores, ficou compreendido entre - 1,01 a 4,94, sendo os valores maiores os que representam os setores com maior vulnerabilidade.

Os dados encontrados para a vulnerabilidade social foram sobrepostos com os encontrados para a vulnerabilidade ambiental, resultando através do cruzamento dos grupos, tendo como resultado final seis níveis de vulnerabilidade socioambiental: muito alta; alta; 
média a alta; média a baixa; baixa e muito baixa; que podem ser representadas através de uma matriz.

Os resultados são apresentados através de mapas com a justaposição dos índices de vulnerabilidade social e o de vulnerabilidade ambiental, indicando com as cores para cada nível o grau da vulnerabilidade socioambiental ao longo do espaço urbano estudado, mostrando ser uma metodologia de fácil visualização dos resultados, apesar dos longos cálculos para se chegar aos índices.

\section{Metodologia de Hogan}

0 estudo de Hogan (2007) foi desenvolvido no contexto urbano de Campinas-SP, sob o título A vulnerabilidade social no contexto metropolitano: o caso de Campinas. A intenção era aprofundar estudos e pesquisas para problemas urbanos que envolvessem as relações da população com o ambiente, e na busca do entendimento dos condicionantes - além da pobreza, da diferenciação das pessoas ou famílias -, estudar a inabilidade de resposta diante dos riscos.

Nesse trabalho, 0 autor parte da hipótese de que há uma coincidência entre espaço suscetível a processos naturais perigosos e os espaços da cidade que apresentam os piores indicadores sociais, econômicos e de acesso aos serviços e infraestrutura urbana, trabalhando numa linha moderada para analisar as relações da dinâmica demográfica, em toda sua complexidade, com a mudança ambiental, considerando importante romper os limites impostos pela questão da população e restringir ou não o progresso, pois outros aspectos mais importantes devem ser considerados nessa dinâmica, buscando uma multidisciplinaridade que envolve o tema da população e meio ambiente.

0 trabalho parte de uma tentativa de sistematizar algumas conclusões a respeito do sentido e da importância do conceito de vulnerabilidade para os estudos urbanos, para, em seguida, buscar sua aplicação empírica a partir do uso de dados secundários, no caso, o censo demográfico de 2000, apresentando como resultado uma divisão da cidade em "zonas de vulnerabilidade", cuja importância reside na possibilidade de identificar, no âmbito intraurbano, carências ou vantagens diferenciadas que, mais além das disponibilidades materiais, possam dar maior poder de resposta ao conjunto de dificuldades que a cidade desigual impõe a seus habitantes.

Para desenvolvimento de seu estudo, Hogan vai abordar os conceitos desenvolvidos por Katzman (1999): capital físico - envolvendo todos os meios essenciais para a busca de bem-estar; capital social - inclui as redes de reciprocidade, confiança, contatos e acesso à informação; e capital humano - que inclui o trabalho como ativo principal e o valor a ele agregado pelos investimentos em saúde e educação, os quais implicam maior ou menor capacidade física para o trabalho. Dessa forma ele classifica seus indicadores de acordo com essa nomenclatura.

Para cada uma dessas três dimensões de indicadores, foram realizadas análises fatoriais, a partir das quais foram obtidos cinco fatores: dois para o capital físico, um para o capital humano e dois para o capital social. Através da interpretação dada aos fatores identificados, resultante de análise fatorial, é que se poderá analisar e interpretar os resultados obtidos, 
particularmente os que se referem aos escores fatoriais assumidos por cada uma das áreas de ponderação, chegando ao seguinte resultado: quanto maior o valor de seu escore, ou seja, quanto mais próximo de 1, piores serão as condições relativas ao fator do indicador, na "área de ponderação".

Essa metodologia apresenta seus resultados através de mapas cartográficos sobreposicionando as áreas de vulnerabilidade social às de riscos ambientais, considerando os fatores quantificados pela metodologia.

\section{Metodologia de Deschamps}

Deschamps $(2004,2006)$ trabalhou inicialmente em sua tese com a vulnerabilidade socioambiental na cidade de Curitiba em 2004. No entanto, seu trabalho mais significativo nessa área corresponde a um estudo desenvolvido pelo Grupo de Pesquisa Observatório das Metrópoles, que comparava a vulnerabilidade socioambiental nas metrópoles brasileiras: São Paulo, Rio de Janeiro, Belo Horizonte, Porto Alegre, Brasília, Curitiba, Recife, Fortaleza, Campinas, Manaus, Vitória, Goiânia, Belém, Florianópolis, Natal e Maringá. Desenvolvido entre 2004 e 2009, esse estudo integrou 0 Projeto Território, Coesão Social e Governança Democrática, financiado pelo $\mathrm{CNPq}$ - Conselho Nacional de Desenvolvimento Científico e Tecnológico, sob a coordenação do professor Dr. Luiz César de Queiroz Ribeiro.

Essa pesquisa está inserida no campo teórico do meio ambiente e desenvolvimento, e apresenta os procedimentos para a construção de tipologias de áreas intraurbanas nas Regiões Metropolitanas brasileiras, avançando na determinação de espaços marcados, por abrigar grupos populacionais socialmente vulneráveis e expostos a situações de risco.

Nesse trabalho, a autora parte da hipótese de que a intensa mobilidade intraurbana faz com que os deslocamentos populacionais, principalmente de grupos populacionais de baixa renda, atinjam áreas sujeitas a riscos ambientais. Assim, parte de uma abordagem que enfatiza a dimensão social dos problemas ambientais e considera as famílias ou pessoas morando numa mesma área como unidade de referência para o desenvolvimento do estudo.

Para o desenvolvimento desse trabalho foram utilizados somente dados secundários disponibilizados pelo IBGE no Censo Demográfico de 2000, com informações das unidades geográficas formadas por agrupamento mutuamente exclusivo de setores censitários, obedecendo aos seguintes critérios: 1) tamanho, em termos de domicílios e população; 2) contiguidade, garantindo o sentido geográfico; e 3) homogeneidade, em relação a um conjunto de características populacionais e de infraestrutura conhecida.

A metodologia trabalha com três dimensões: econômica, social e ambiental, abordadas na perspectiva de que o risco é um aspecto negativo e causador de danos a determinados segmentos sociais. Ela mensura a dimensão ambiental através da inadequação dos indicadores urbanos, considerando a ausência combinada dos três serviços básicos esgotamento sanitário por rede geral ou fossa séptica, água canalizada em pelo menos um cômodo e coleta de lixo. Ela justifica a escoIha desses indicadores, salientando que eles são fatores que afetam a qualidade de vida numa perspectiva saudável, sabendo que a 
ausência de condições adequadas de saneamento tem importante rebatimento na proliferação de doenças.

A tipologia e o agrupamento das áreas das Regiões Metropolitanas foram obtidos por dois métodos estatísticos multivariados: análise fatorial por componentes principais e análise de agrupamento. A análise fatorial por agrupamentos avalia as intercorrelações entre variáveis, com o objetivo de identificar um menor número de fatores que apresentem aproximadamente o mesmo total de informações expresso pelas variáveis originais. $\mathrm{Na}$ análise por componentes principais, calculam-se os autovalores e a matriz de correlação entre variáveis originais e os fatores comuns.

Como resultado, a metodologia apresenta um quadro da vulnerabilidade socioambiental por meio da leitura cruzada da vulnerabilidade social e risco ambiental, mostrando o resultado em quatro quadrantes, classificados em: 1) combinação de baixa vulnerabilidade social com baixo risco ambiental; 2) combinação de baixa vulnerabilidade social com alto risco ambiental; 3) combinação de alta vulnerabilidade social com baixo risco ambiental; e 4) combinação de alta vulnerabilidade social com alto risco ambiental.

Os modelos apresentados neste estudo apontam pontos semelhantes e diferentes nas abordagens que são significativos e determinísticos para a construção de um quadro de avaliação da vulnerabilidade socioambiental nos espaços urbanos. Dessa forma, cabe especificar, entender o campo teórico e os fenômenos estudados, para comparar os fundamentos necessários para uma adaptação à realidade das diversidades urbanas específicas do caso brasileiro.

\section{Comparativo das metodologias de vulnerabilidade socioambiental}

Devido à conjuntura política e socioeconômica praticante na maioria nos países latino-americanos, os estudos que envolvem a vulnerabilidade socioambiental têm considerado os aspectos socioeconômicos como mais importantes que os ambientais. Dessa forma, Blaikie et al. (1996) afirmam que as questões de raça, sexo, idade, educação, renda e situação de trabalho são determinísticas para a condição de vulnerabilidade, porque incidem diretamente no poder de resiliência da população.

Os aspectos ambientais, nesse contexto geográfico latino-americano, são refletidos na perspectiva do adensamento populacional e da dinâmica urbana das grandes cidades, que, devido à interferência dos processos de urbanização, direcionam essa camada pobre para as áreas periféricas ou de proteção ambiental, como rios e encostas. Não se pode, entretanto, descartar alguns fenômenos naturais específicos de alguns países latino-americanos como terremotos, nevascas, vulcões, furacões, dentre outros, que de modo esporádico e cíclico ocasionam danos.

Segundo Abramovay (2002), os estudos ancorados na vulnerabilidade na América Latina foram motivados pela preocupação em abordar de forma mais integral e completa não só os fenômenos da pobreza, mas ainda, as diversas modalidades de desvantagem social. Tais estudos buscaram observar os riscos de mobilidade social que afetam a todos, independentemente de sua classe social, abarcando a vulnerabilidade na dinâmica do bem-estar social atrelada às dimensões associada a esse processo. 
No Brasil a vulnerabilidade é tratada utilizando uma sobreposição de riscos tanto ambientais quanto sociais, considerando que os riscos sociais se relacionam com aspectos ligados, dentre outros, a dinâmica social, segregação urbana, injustiças ambientais - os vulneráveis como vítimas de uma proteção desigual -, enquanto os ambientais são relacionados às ameaças naturais ocorrentes em áreas específicas.

Nessa perspectiva teórica, os modelos brasileiros que estudaram a vulnerabilidade socioambiental mostraram uma evolução em sua sistematização e ferramentas ao longo do tempo. Observa-se que os pioneiros como Hogan e Deschamps abriram caminhos para esse tipo de estudo, trabalhando suas metodologias com enfoque teórico próprio, se mostrando precursores da abordagem.

Consciente da importância desses estudos para o desenvolvimento e aprimoramento das pesquisas que envolvem a vulnerabilidade socioambiental, serão analisados cinco modelos, comparando os aspectos metodológicos e seus campos teóricos, buscando uma aproximação entre os mesmos. Considerando seis aspectos para análise: 1) quanto à característica do método; 2) quanto ao campo geográfico de atuação; 3) quanto ao foco das dimensões mensuradas; 4) quanto às variáveis trabalhadas; 5) quanto ao tratamento dos dados; 6) quanto ao vínculo com grupos de pesquisas.

\section{Características do método}

Os cinco modelos analisados adotam abordagem dedutiva, em que são testadas as premissas construídas a partir de pressupostos derivados de um marco teórico consistente, testando-os, coletando dados apropriados e explorando as relações entre medidas que operacionalizam tais conceitos.

Dessa forma, os modelos partem em sua totalidade, do pressuposto de que o processo de expansão urbana impulsiona a fixação de famílias ou grupos populacionais em áreas ambientalmente inapropriadas, expondo-as a situações constantes de vulnerabilidade, porque esses grupos ficam sem condições socioeconômicas de resposta diante das vulnerabilidades e dos desastres ambientais.

\section{Campo geográfico de atuação}

O campo geográfico dos modelos analisados por Almeida (2010), Hogan (2007) e Deschamps (2004) diz respeito a áreas metropolitanas, se caracterizando como um estudo local focalizado em áreas intraurbanas, buscando a articulação espacial com a economia, a política, a cultura e a dominação socioespacial que o centro urbano exerce sobre o restante da cidade.

No estudo de Alves (2010a), a análise se foca no Distrito, reduzindo a área geográfica estudada para uma região dentro do espaço urbano - podendo ser chamada de microrregião -, trabalhando sobre o aspecto das tipologias de utilização do solo em áreas urbanas, tais como: favelas; conjuntos habitacionais (unifamiliares e multifamiliares) e áreas industriais/comerciais/institucionais. Analisa, em escala intraurbana, as interrelações entre processos de expansão urbana e situações de vulnerabilidade socioambiental. 
Esse modelo pode ser analisado tomando-se como referência as teorias de Lefebvre (1991), que trata da segregação espacial, procurando, em um esforço analítico e empírico, uma forma de entender esse fenômeno sob três prismas, ora sucessivos, ora simultâneos: espontâneos (provenientes das rendas e ideologias) - voluntário (estabelecendo espaços separados) - programado (sob pretexto de arrumação e plano). No entanto, percebe-se que, apesar de a pesquisa estudar os espaços urbanos produzidos a partir da égide capitalista, em que existe uma intencionalidade de ocupação do solo urbano por diferentes camadas sociais, não enfatiza os processos sociais, nem econômicos que vulnerabilizam a população, como também não relevam na mobilidade social urbana os motivos que trouxeram essa população para esse tipo de ocupação.

Dessa maneira, os aspectos relacionados por Cardona (1997), Abramovay (2002), Lavinas (2002), relativos à exclusão e vulnerabilidade social, não são tratados de forma direta, e, sim, incorporados às tipologias urbanas existentes na cidade, servindo apenas como meio de entender a ocupação urbana sob os aspectos únicos da expansão urbana desassociados dos processos socioeconômicos que vulnerabilizam a população da cidade de Tiradentes.

Alves (2010b) trabalha com 16 cidades da faixa litorânea de São Paulo, buscando analisar a relação entre a vulnerabilidade ambiental, causada pelas mudanças climáticas, e o processo de apropriação do espaço urbano, pelos grupos populacionais mais vulneráveis socialmente. As variáveis ambientais consideradas nesse estudo partem de uma análise baseada nos efeitos ocasionados à natureza pela ação antrópica. Comparando esse estudo com o de Micklin (1999), que inclui a degradação ambiental urbana, entre os grandes desafios ambientais para a América Latina, observa-se uma semelhança nas preocupações, mas uma distinção de enfoque por considerar como responsabilidade única da degradação a ação do homem, que invade áreas de preservação, não considerando, assim, as responsabilidades políticas e institucionais que levam o homem a essa invasão.

Considerando as teorias de Liverman (1990), o estudo de Alves (2010b) concentra esforços na caracterização da população sujeita a risco de desmoronamentos, e considera a vulnerabilidade como risco do lugar - conceito de Cutter (2003) - e como geograficamente centrada, mas com efeitos diferentes de acordo com a capacidade de resposta da população.

Observa-se que todos os modelos, apesar do afunilamento do campo geográfico utilizado por Alves (2010a; 2010b), analisam as áreas urbanas baseados na apropriação do espaço por grupos sociais, no processo de transbordamento urbano, considerando as áreas periféricas e periurbanas.

\section{Dimensões mensuradas}

Os estudos latino-americanos que envolvem a vulnerabilidade socioambiental, tais como Barrenechea (2000), Moser (1998), Gonzáles (1998), Blaikie et al. (1996), D’Ercole (1994) estabelecem que a vulnerabilidade é uma combinação de características de um grupo social derivada de suas condições sociais e econômicas relacionadas a uma periculosidade específica. Dessa forma, a vulnerabilidade socioambiental precisa interrelacionar a dimensão 
socioeconômica da população à dimensão ambiental dos fenômenos naturais, os quais podem ameaçar determinados grupos sociais.

Dos cinco modelos estudados, três trabalham com duas dimensões: socioeconômica e ambiental. Em Alves (2010b), Almeida (2010) e Deschamps (2004), as dimensões valorizadas recaem no risco socioeconômico, que afligem grupos ou famílias, sendo as variáveis sociais tratadas, através de indicadores como: demografia, anos de escolaridade, reprodução, questão etária, de gênero e quantidade de dependentes; sendo as variáveis econômicas tratadas através de indicadores relativos à renda e situação de emprego, os quais estão muitas vezes correlacionados com a questão educacional, e, por fim, os indicadores referentes a vulnerabilidade ambiental, que recaem sobre a inadequação construtiva, aspectos de propriedade do imóvel e aspectos da infraestrutura urbana referentes ao abastecimento de água, esgoto e coleta de lixo.

0 modelo de Hogan (2007) não trabalha com dimensões, e, sim, com o conceito de capital social, humano e físico desenvolvido por Kaztman (1999). Alves (2010a) trabalha com a vulnerabilidade partindo das tipologias urbanas, considerando os assentamentos precários - favelas e loteamentos irregulares como aqueles com maior vulnerabilidade socioambiental devido às características da população que se alojam nesses espaços - assim prioriza os aspectos urbanísticos tratados nos estudos de D’Ercole (1994).

Os modelos de Alves (2010b) e Almeida (2010) se diferenciam dos outros, porque, no aspecto das dimensões ambientais, vão enfatizar as questões que são consequentes dos riscos relativos às mudanças climáticas. Assim, Alves (2010b) trabalha com os riscos de desmoronamento e Almeida (2010), com enchentes, se aproximando dos estudos de Barrenechea (2000) e Gonzáles (1999), as quais trataram desses mesmos aspectos na Argentina.

Apesar das especificidades na abordagem dos cinco modelos estudados observa-se que a importância dos indicadores da dimensão socioeconômica recai no pressuposto de que, na sociedade moderna, determinadas características das famílias limitam a acumulação de recursos. Nesse viés, os indicadores sociais muitas vezes determinam o nível de qualidade econômica da família, porque criam condições de concorrência para o mercado de trabalho. Assim, as famílias chefiadas por analfabetos, mulheres, idosos ou adolescentes, estariam em maior vulnerabilidade do que famílias chefiadas por pessoas com nível educacional mais alto, do gênero masculino ou numa faixa etária adulta, porque pressupõem condições de rendimento e trabalho melhores, ratificando os estudos produzidos por Lavell (2005), Abramovay (2002) e Blaikie et al. (1996).

Outro aspecto a considerar são os fatores que indicam a vulnerabilidade sociodemográfica relacionada à quantidade de filhos, agregados, presença de idosos, de jovens e adolescentes. Esse fator mostra que existe uma forte correlação com as desvantagens socioeconômicas as quais pressupõem pobreza, baixos rendimentos, informalização do 
trabalho, não sequência escolar e condições inadequadas de moradia.

Os processos de exclusão social fragilizam a população de pobres e miseráveis e influem na capacidade de respostas dos indivíduos diante de situação de risco. Nessa perspectiva, os aspectos sociais da vulnerabilidade são de grande importância em um país onde as injustiças sociais prevalecem (Abramovay, 2002; Lavinas, 2002; Busso, 2001), onde os direitos de muitos são colocados em segundo plano para favorecer uma minoria, confirmando a necessidade de considerar a dimensão social da vulnerabilidade em nosso país. Dessa forma, pode-se quantificar o nível de utilização da dimensão social da vulnerabilidade socioambiental no Brasil, dada pelos modelos estudados, através do Gráfico 1.
A quantidade em maior número de indicadores sociais e sociodemográficos - $77 \%$ - refletem diretamente nos indicadores econômicos, estabelecendo uma relação biunívoca de causa e efeito, pois as famílias economicamente vulneráveis precisam que jovens contribuam financeiramente e relevem a educação para um segundo plano, criando assim um círculo vicioso de causa-efeito na propagação da vulnerabilidade das famílias.

Dessa forma, os indicadores econômicos - 13\% - utilizados nas metodologias estão, assim, relacionados com outros aspectos da vulnerabilidade relativos ao mercado de trabalho, ou seja, refletindo a conjuntura econômica do país, como pessoas sem carteira de trabalho assinada, condição da desigualdade de gênero, informalidade do trabalho, renda não proveniente do trabalho e baixos salários.

\section{Gráfico 1 - Percentual de indicadores por dimensões trabalhadas nos cinco modelos}

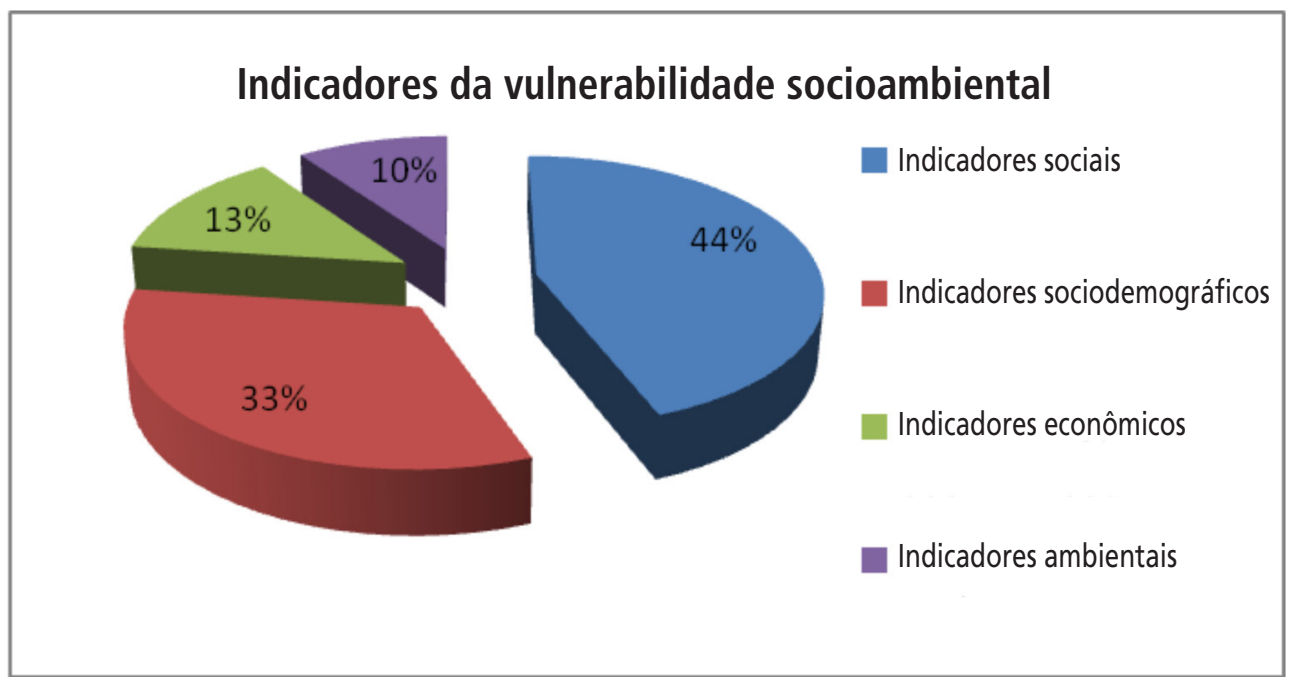

Fonte: Elaboração própria (2013). 
Na dimensão ambiental, a importância dos indicadores recai nos fenômenos ambientais que foram intensificados com as mudanças climáticas. No caso do Brasil, alguns fenômenos não fazem parte desse repertório, até o momento, como furacões, tornados, terremotos, vulcões, dentre outros. No entanto, o Brasil é um país rico em bacias hidrográficas e em topografia, o que gera grandes riscos de enchentes, deslizamentos, desmoronamentos e vendavais.

Diante dessa realidade, duas metodologias abarcaram esses aspectos, buscando aprofundar o nível que as vizinhanças próximas a esses locais sofrem com esses riscos. Alves (2010b) vai trabalhar com os riscos de deslizamentos, buscando os indicadores de altimetria e declividade, e Almeida (2010) vai trabalhar com os riscos de enchentes, através do tempo de retorno das cheias dos rios.

A metodologia de Alves (2010a) buscou, nas tipologias urbanas, o viés da vulnerabilidade socioambiental, quando associou essas tipologias - assentamentos precários, não precários e áreas urbanizadas - aos aspectos econômicos e de qualidade de vida das famílias moradoras do lugar, fazendo a análise e considerando apenas os mapas cartográficos.

Em nenhum dos modelos explorados são utilizadas variáveis que contemplem, em um único modelo, indicadores mais completos relativos à dimensão ambiental, mesmo sabendo-se das condições geográficas brasileiras ricas em relevo e rios. Esses modelos contrariam as teorias de Torres e Marques (2001) que consideram que a vulnerabilidade só pode ser vista em sua plenitude quando superpondo, em termos espaciais, os indicadores socioeconômicos, com os riscos ambientais e serviços assistenciais, os quais podem nos fornecer parâmetros para apontar certa deficiência nas abordagens feitas nos cinco modelos, por não considerarem em seu conjunto de indicadores esses aspectos citados, além de outros, que influenciam o processo de vulnerabilidade.

Considerando o conjunto de indicadores que compõem a dimensão ambiental, pode-se comprovar que houve uma deficiência nas abordagens, pois apenas $10 \%$ dos indicadores se encontravam nessa dimensão (ver Tabela 1), destacando que esses indicadores só foram encontrados em duas metodologias das cinco estudadas, as quais buscavam objetivos diferentes nas pesquisas. Os vendavais não foram considerados em nenhuma pesquisa, apesar da maioria das pesquisas serem desenvolvidas nas regiões Sul e Sudeste, onde a incidência de vendavais precisa ser considerada, pois fazem parte do repertório de risco.

Autores como Vignoli (2000), Camarano e Gahouri (1999) e Moser (1998) discutem a possibilidade de uma ciência multidisciplinar da vulnerabilidade, que possa abarcar diferentes formas de risco a que a sociedade está exposta, cujas conexões entre elas formam uma malha de causa e efeito, uma sobre a outra, defendendo que não se pode mais analisar a vulnerabilidade sobre uma dimensão somente. Diante dessa afirmação, nota-se a fragilidade das cinco metodologias aqui expostas, que não consideram os aspectos ambientais como determinísticos no processo de vulnerabilidade. Esse estudo reconhece que, no país com tantas desigualdades sociais, os aspectos socioeconômicos são preponderantes, mas que os aspectos ambientais também exercem grande influência para tal processo, pois servem como um gatilho agravador da vulnerabilidade socioambiental. 
As características dos indicadores feitas pelos autores seguiram os seguintes pressupostos: a) utilizam variáveis mensuráveis; $b$ ) são significativos para o enfoque do estudo; c) são relevantes para as decisões que orientam as políticas públicas; d) são de fácil comunicação e interpretação; e) permitem um enfoque integrado e sistêmico; f) são de fácil obtenção. Além desses critérios, observa-se, ainda, que a diversidade dos aspectos que envolvem a vulnerabilidade leva à necessidade de abordagens das dimensões e indicadores de forma abrangente e integrada.

\section{Variáveis trabalhadas}

Quatro modelos trabalham com dois tipos de variáveis. Uma independente, que se refere ao processo de expansão urbana que impulsiona a população a se fixar em áreas impróprias a moradia; e outras dependentes relativas às questões socioeconômicas e ambientais relacionadas com as famílias ou grupos sociais que estão condicionadas à variável independente.

Observa-se uma correlação dessas variáveis trabalhadas nos cinco modelos brasileiros com os estudos feitos por D'Ercole (1994), o qual incorporou em seus estudos sobre a vulnerabilidade urbana fatores inerentes ao crescimento populacional que interagem com a dinâmica urbana.

\section{Tratamento de dados}

Os dados são tratados através de duas ferramentas, uma de base estatística e a outra através da construção e análise de mapas georreferenciados que especificam as situações ambientais e socioeconômicas da população estudada.

$\mathrm{Na}$ análise estatística dos indicadores sociais e econômicos, em todos os modelos foram executados três procedimentos: o primeiro, de análise multivariada para escolher quais as variáveis socioeconômicas e sociodemográficas seriam mais relevantes para estabelecer uma tipologia das áreas estudadas; a segunda, de análise fatorial, estudando as intercorrelações internas de um conjunto de variáveis; e a terceira, buscando uma sumarização dos dados - foi aplicado o método do agrupamento não hierárquico das k-médias para fazer uma análise comparativa dos resultados.

As análises das variáveis ambientais são feitas baseadas em mapas georreferenciados, que estabelecem as áreas urbanas que apresentam riscos ambientais, utilizando programas computacionais específicos para cada caso. Em seguida, os resultados dos dois procedimentos são cruzados, estabelecendo as áreas que apresentam a vulnerabilidade social cruzada com a ambiental no mesmo contexto.

\section{Vínculo com grupos de pesquisas}

Todos os cinco modelos apresentados fazem parte de grupos de pesquisas consolidados e atuantes no Brasil. Alves (2010a; 2010b) está vinculado ao Centro de Estudos da Metrópole (CEM-Cebrap); Almeida (2010) está vinculado ao grupo de pesquisa da UFRN, Dinâmicas Ambientais, Riscos e Ordenamento do Território; Hogan (2007) ao Núcleo de Estudos Populacionais (Nepo) e Deschamps (2004; 2006) ao Observatório das Metrópoles (ver Quadro 1). 
Quadro 1 - Comparativo dos Modelos

\begin{tabular}{|c|c|c|c|c|c|}
\hline \multirow{2}{*}{ Características } & \multicolumn{5}{|c|}{ Modelos } \\
\hline & Alves (a) & Alves (b) & Almeida & Hogan & Deschamps \\
\hline $\begin{array}{l}\text { Características } \\
\text { do método }\end{array}$ & Dedutivo & Dedutivo & Dedutivo & Dedutivo & Dedutivo \\
\hline $\begin{array}{l}\text { Campo geográfico } \\
\text { de atuação }\end{array}$ & $\begin{array}{l}\text { Micro urbano } \\
\text { (distrito) }\end{array}$ & $\begin{array}{l}\text { Micro urbano } \\
\text { (litoral) }\end{array}$ & Cidade & Cidade & Metrópoles \\
\hline $\begin{array}{l}\text { Dimensões } \\
\text { mensuradas }\end{array}$ & $\begin{array}{l}\text { Socioeconômica } \\
\text { e ambiental }\end{array}$ & $\begin{array}{l}\text { Socioeconômica } \\
\text { e ambiental }\end{array}$ & $\begin{array}{l}\text { Socioeconômica } \\
\text { e ambiental }\end{array}$ & $\begin{array}{c}\text { Capital físico, social } \\
\text { e humano }\end{array}$ & Socioeconômica \\
\hline Variáveis trabalhadas & $\begin{array}{c}\text { Variáveis embutidas } \\
\text { nas tipologias } \\
\text { urbanas }\end{array}$ & $\begin{array}{l}\text { Variável } \\
\text { independente } \\
\text { e dependente }\end{array}$ & $\begin{array}{l}\text { Variável } \\
\text { independente } \\
\text { e dependente }\end{array}$ & $\begin{array}{l}\text { Variável } \\
\text { independente } \\
\text { e dependente }\end{array}$ & $\begin{array}{c}\text { Variável } \\
\text { independente } \\
\text { e dependente }\end{array}$ \\
\hline Tratamento de dados & Georreferenciamento & $\begin{array}{c}\text { Georreferenciamento } \\
\text { e estatística }\end{array}$ & $\begin{array}{c}\text { Georreferenciamento } \\
\text { e estatística }\end{array}$ & $\begin{array}{c}\text { Georreferenciamento } \\
\text { e estatística }\end{array}$ & $\begin{array}{c}\text { Georreferenciamento } \\
\text { e estatística }\end{array}$ \\
\hline $\begin{array}{c}\text { Vínculo com grupos } \\
\text { de pesquisas }\end{array}$ & CEM/Cebrap & CEM/Cebrap & $\begin{array}{l}\text { Dinâmicas } \\
\text { ambientais, riscos } \\
\text { e ordenamento do } \\
\text { território/UFRN }\end{array}$ & Nepo/Nesur & $\begin{array}{l}\text { Observatório das } \\
\text { Metrópoles }\end{array}$ \\
\hline
\end{tabular}

Fonte: Elaboração própria (2012).

Dessa forma, observa-se que os modelos apresentados foram contemplados com conhecimentos acadêmicos de seus autores, que trouxeram suas experiências profissionais para o desenvolvimento das teorias e aplicações feitas em suas pesquisas, contribuindo para a elaboração do campo teórico e prático da vulnerabilidade socioambiental brasileira.

\section{Conclusões}

Os cinco modelos foram de grande importância para servir de base aos estudos da vulnerabilidade socioambiental no Brasil, permitindo um progresso através do uso de novas técnicas e ferramentas, as quais possibilitaram um diagnóstico favorável dos fatores que contribuíam para vulnerabilidade socioambiental no contexto brasileiro, em cada especificidade territorial e temporal considerada.
A dimensão socioeconômica, nos cinco estudos, foi bem abordada contemplando os principais aspectos que podem aumentar o processo de vulnerabilidade das famílias brasileiras, sendo um caminho de diagnóstico e podendo ser abordada em qualquer localidade geográfica brasileira, pois contempla a realidade socioeconômica comum em todos os estados e cidades, pois esse tipo de problema é consequência de uma situação genérica nacional, ou seja, de falta de políticas públicas nacionais voltadas para um planejamento em longo prazo que busque erradicar a pobreza de forma nacional e integrada.

Esta pesquisa assinala para a necessidade de uma evolução nos procedimentos e dimensões utilizados, se justificando pela necessidade de uma padronização sistemática que permita uma análise mais profícua sobre os aspectos específicos a serem considerados sobre nossa realidade, não devendo ser descartada a 
evolução dos estudos anteriores feitos por esses pesquisadores.

Assim, observa-se a necessidade de aprimoramento da dimensão ambiental, tratada de forma superficial nos estudos analisados, e a incorporação de outras variáveis para abarcar outras dimensões não contempladas nesses cinco modelos estudados. Relacionada às variáveis ambientais, existe a necessidade de considerar os indicadores relacionados à exposição de risco naturais existentes nos espaços urbanos do Brasil: enchentes, desmoronamentos, deslizamentos, vendavais, chuva de granizo e ciclones, pois o estudo direcionado a uma só dimensão do risco ambiental - como os estudados por Alves (2010b) e Almeida (2010) - desconsidera a existência de outras ameaças que podem ocorrer no mesmo contexto geográfico, e, pior ainda, num período temporal próximo, o que acarretaria uma maior vulnerabilidade.

Observou-se a necessidade de incorporação da dimensão psicológica referente à percepção ambiental, a qual foi desconsiderada em todos os modelos brasileiros. Nas referências teóricas, a percepção ambiental é de fundamental importância para os estudos de vulnerabilidade, porque o nível dessa percepção faz diferença em seu poder de mitigação e resiliência diante dos riscos socioambientais a que estão expostos.

A percepção ambiental ajuda na formação cidadã de um sujeito ecológico, a qual exerce uma função importante no exercício da cidadania e aguça a capacidade de enxergar o mundo, quando o sujeito passa a pautar suas atitudes e suas ações dentro de uma visão de mundo baseada em princípios socioambientais, o qual estabelece amplo conjunto de práticas proativas para a conservação da natureza e melhoria da qualidade de vida.

A dimensão política institucional também se apresenta como de grande importância para o contexto da vulnerabilidade socioambiental no Brasil, porque identifica as ações políticas e administrativas de contenção aos fatores de riscos que ameaçam as populações, servindo como termômetro para mensurar as políticas públicas necessárias para o suprimento das necessidades sociais, econômicas, ambientais e como resposta às revindicações feitas pela sociedade civil.

Outro aspecto negligenciado, nos estudos analisados, é a especificidade da situação de vulnerabilidade, pois grupos populacionais podem estar sujeitos ao mesmo perigo, mas não apresentem o mesmo risco, por não estarem igualmente em situação de vulnerabilidade, assim os indicadores devem ser levantados através de dados primários que identificam, no lugar e tempo, as fragilidades das famílias que estão expostas aos riscos.

Observa-se que os modelos utilizados abrem espaço para a elaboração de novas abordagens, no entanto, deixam a desejar, porque não conseguem abranger outras variáveis que são importantes para o contexto geográfico e cultural brasileiro, e, quando aplicadas de forma limitada, não conseguem contemplar os reais riscos existentes. 


\section{Mônica Maria Souto Maior}

Instituto Federal de Educação, Ciência e Tecnologia da Paraíba, Unidade Acadêmica de Infraestrutura. João Pessoa/PB, Brasil.

mmsmaior@hotmail.com

\section{Gesinaldo Ataíde Cândido}

Universidade Federal de Campina Grande, Programa de Pós-graduação em Recursos Naturais. Campina Grande/PB, Brasil. gacandido@uol.com.br

\section{Referências}

ABRAMOVAY, M. et al. (2002). Juventude, violência e vulnerabilidade social na América Latina: desafios para políticas públicas. Brasília, Unesco-BID.

ALMEIDA, L. Q. de (2010). Vulnerabilidade socioambiental de rios urbanos: bacia hidrográfica do Rio Maranguapinho região metropolitana de Fortaleza-Ceará. Tese de doutorado. Rio Claro, Universidade Estadual Paulista "Júlio de Mesquita Filho".

ALVES, C. D. et al. (2008). Análise dos processos de expansão urbana e das situações de vulnerabilidade socioambiental em escala intra-urbana. In: IV ENCONTRO NACIONAL DA ANPPAS. Anais. Brasília.

ALVES, H. P. et al. (2010a). Dinâmicas de urbanização na hiperperiferia da metrópole de São Paulo: análise dos processos de expansão urbana e das situações de vulnerabilidade socioambiental em escala intraurbana. Revista Brasileira de Estudos Populacionais. Rio de Janeiro, v. 7, n. 1, pp. 141-159.

(2010b). Vulnerabilidade socioambiental nos municípios do litoral paulista no contexto das mudanças climáticas. In: XVII ENCONTRO NACIONAL DE ESTUDOS POPULACIONAIS. Anais. Caxambu.

BARRENECHEA, J. et al. (2000). Una propuesta metodológica para el studio de la vulnerabilidad social en el marco de la teoria social del riesgo. In: IV JORNADAS DE SOCIOLOGIA FACULDAD DE CIENCIAS SOCIALES-UBA. Anais. Buenos Aires.

BLAIKIE, P. M.; CANNON, T.; DAVIS, I. e WISNER, B. (1994). Atrisk: natural hazards, people's vulnerability, and disasters. London, Routledge.

(1996). Vulnerabilidad el entorno: social, político y económico de los desastres. Puerto Limón, Costa Rica. LA RED - Read de Estudios Sociales en Prevencion de Desastres em América Latina.

BUSSO, G. (2005). Pobreza, exclusión y vulnerabilidad social: usos, limitaciones y potencialidades para el diseno de políticas de desarrollo y de población. Santiago do Chile, Cepal/Celade.

CAMARANO, A. A. e GAHOURI, S. (1999). "Idosos brasileiros: que dependência é essa?” In: CAMARANO, A. A. (org.). Muito além dos 60: os novos idosos brasileiros. Rio de Janeiro, Ipea. 
CARDONA, O. D. (1996). “Manejo ambiental y prevención de desastres”. FERNÁNDEZ, M. A. (org.). Cities at Risk. Puerto Limón, Costa Rica, LA RED/USAID.

CASTEL, R. (1997). A dinâmica dos processos de marginalização: da vulnerabilidade à "desfiliação". Cadernos Centro de Recursos Humanos-CRH. Salvador, n. 26, pp. 19-40.

CUTTER, S. L. (2003). Vulnerability to environmental hazards. Progress in human geography. Los Angeles, v. 20, n. 4, pp. 529-539.

D’ERCOLE, R. (1994). Les vulnérabilités des sociétés et des espaces urbanisés: concepts, typologie, modes d'analyse. Revue de Géographie Alpine. Paris, v. 82, n. 4, pp. 87-96.

DEPARTAMENTO INTERSINDICAL DE ESTATÍSTICA E ESTUDOS SOCIOECONÔMICOS - DIEESE (2007). Aspectos conceituais da vulnerabilidade social. Projeto de qualificação social para atuação de sujeitos ou grupos sociais na negociação coletiva e na gestão de políticas públicas. Convênio MTE/SPPE/CODEFAT - n. 075/2005 e Primeiro Termo Aditivo.

DESCHAMPS, M. V. (2004). Vulnerabilidade socioambiental na região metropolitana de Curitiba. Tese de doutorado. Paraná, Universidade Federal do Paraná.

(2006). Vulnerabilidade socioambiental nas regiões metropolitanas brasileiras. Brasília, Relatório de atividades do Observatório das Metrópoles. Convênio Ministério das Cidades/ Observatório das Metrópoles/Fase/Ipardes.

FOURNIER, d'A. E. M. (1995). The quantification of seismic hazard for the purposes of risk assessment. In: INTERNATIONAL CONFERENCE ON RECONSTRUCTION, RESTAURATION AND URBAN PLANNING OF TOWNS AND REGIONS IN SEISMIC PRONE AREAS. Anais. Skopje.

GONZÁLES, S. et al. (1999). Riesgos en Buenos Aires. Caracterización preliminar. In: SEMINARIO DE INVESTIGACIÓN EL NUEVO MILENIO Y LO URBANO. Anais. Buenos Aires.

HEWITT, K. (1997). Regions of risk. Harlow, Longman.

HOGAN, D. J. et al. (orgs). (1999). Migração e ambiente nas aglomerações urbanas. Campinas, Nepo/ Unicamp.

KAZTMAN, R. et al. (1999). Vulnerabilidad, activos y exclusión social en Argentina y Uruguay. Santiago do Chile, OIT. (Documento de trabalho, pp. 107).

KAZTMAN, R. (2000). Notas sobre la medición de la vulnerabilidad social. México: BID-BIRF-Cepal. Borrador para discusión. 5 Taller regional, la medición de la pobreza, métodos e aplicaciones. Disponível em: http://www.eclac.cl/deype/ noticias/proyectos. Acesso em: 2 jun 2012.

KOWARICK, L. (2003). Sobre a vulnerabilidade socioeconômica e civil: Estados Unidos, França e Brasil. Revista Brasileira de Ciências Sociais - RBCS. São Paulo, v. 18, n. 51, pp. 61-86.

LAVELL, A. (2000). “Desastres y desarrollo: hacia un entendimiento de las formas de construcción social de un desastre: el caso del Huracán Mitch em Centroamérica". In: GARITA, N. e NOWALSKI, J. (orgs.). Del desastre al desarrollo sostenible: Huracán Mitch en Centroamérica. BID-CIDHCS.

LAVINAS, L. (2002). Pobreza e exclusão: traduções regionais de duas categorias da prática. Econômica. Rio de Janeiro, v. 4, n. 1, pp. 25-59.

LEFEBVRE, H. (1991). The production of space. Oxford, Blackwell. 
LIVERMAN, D. (1990)."The regional impact of global warming in Mexico: incertainty, vulnerability and response". In: SCHMANDT, J. e CLACKSON, J. (orgs.). The regions of global warming: impacts and response strategies. Nova York, Oxford University Press.

MICKLIN, M. (1999). "The ecological transition in Latin American and the Caribbean: theoretical issues and empirical patterns". In: BILSBORROW, R. E. e HOGAN, D. J. (orgs.). Population and deforestation in the humid tropics. Liége, IUSSP.

MOSER, C. (1998). La vulnerability framework: reassessing urban poverty reducion stratregies. World development. Grã Bretanha, v. 26, n. 1, pp. 1-19.

SILVEIRA, H. (2010). Estudo da degradação e do impacto socioambiental na Bacia do Córrego Osório, Maringá - Paraná. Revista Geografar. Curitiba, v. 5, n. 1, pp. 176-205. Disponível em: http:// www.ser.ufpr.br/geografar. Acesso em: 6 jun 2012.

TORRES, H. G. e MARQUES, E. (2001). Reflexões sobre a hiperperiferia: novas e velhas faces da pobreza no entorno metropolitano. Revista Brasileira de Estudos Urbanos e Regionais. Rio de Janeiro, v. 17, n. 4, pp. 97-128.

VIGNOLI, J. R. (2000). Vulnerabilidad demográfica: una faceta de las desventajas sociales. Santiago do Chile, Cepal.

Texto recebido em 19/maio/2013

Texto aprovado em 8/ago/2013 